provide a net increase in the overall abundance of threatened species, or reduce the rate of species loss? Waldron et al. now offer a way to tackle this problem of outcome assessment.

The authors' work demonstrates an effective way to estimate the impact per dollar of conservation spending, and to quantify how changes in investment affect biodiversity. They analysed how conservation spending in 109 countries affected biodiversity declines on a national level for these countries between 1996 and 2008. They used an adjustment technique to reflect differences in purchasing power per dollar in different countries, converting money into 'international' dollars.

To assess biodiversity changes, the authors tracked the endangered status of bird and mammal species assigned to risk-of-extinction categories in a standard resource for such global assessments, known as the International Union for Conservation of Nature Red List ${ }^{8}$. By determining the net change in the Red List status of all the birds and mammals in a country over time, and correlating that change with conservation investment, Waldron and colleagues' model demonstrates a statistically significant, positive correlation between how much a country invests in the protection of threatened species and its success in limiting biodiversity declines. This work provides evidence that an increase in direct national investment in conservation actions is essential to achieve globally agreed biodiversity goals.

As well as analysing conservation spending, another key aspect of the author's model is that it incorporates the effects of other facets of human societies that can affect biodiversity, such as human population growth and increases in agricultural land use. Because human activities that can promote or threaten biodiversity are included in the analysis, the results provide a useful perspective, given that many biodiversity studies do not usually take such a comprehensive approach. When the authors tested their model against additional data, the results validated the model's predictive power.

The authors' findings have broad implications for global biodiversity policy. Notably, given predicted patterns of human population growth and other socio-economic changes, the model indicates that achieving a certain level of biodiversity conservation will cost comparatively more in the future (taking inflation into account) than the cost of taking action now. The level of conservation funding is one of the factors most tightly linked to the successful recovery of endangered species. However, a study of funding in the United States revealed that it is both insufficient and allocated in an uneven way between different groups of species ${ }^{9}$. When a strategy for good conservation investment is being chosen, it should, like any other investment strategy, be guided by balancing benefits and risks ${ }^{10-13}$.

Conservation models that are simple and that can be used for forecasting are particularly suited for national-level decision-making. By showing biodiversity outcomes under different economic, agricultural and populationchange scenarios, Waldron and colleagues' model provides a flexible tool for estimating the impacts of different policies, and for finding approaches to resolve the potential conflicts that can arise when trying to achieve different Sustainable Development Goals for example, by determining the increased funding for biodiversity conservation needed to counterbalance the negative consequences for biodiversity that agricultural expansion may incur.

Humans rely on nature in countless ways. As biodiversity declines, we will lose these tangible benefits, as well as opportunities to delight in the beauty of biodiversity itself. Waldron and colleagues' model affirms a frequent theme in evaluations of the effect of expenditure on biodiversity conservation, which is that halting the decline in global biodiversity would be remarkably cheap. For example, the model suggests that investment of an extra 5 million international dollars in Peru, one of the world's most biodiverse nations, would have halved the rate of biodiversity decline in this country during the 1993-2008 period (Fig. 1). Less than $0.01 \%$ of global gross domestic product would be enough to all but stop species extinctions ${ }^{11}$. Small changes in public policy in a few key nations, and some targeted international investment, could dramatically reduce global biodiversity loss.

Hugh P. Possingham is at The Nature Conservancy, South Brisbane, Queensland 4101, Australia, and The University of Queensland. Leah R. Gerber is at the School of Life Sciences and Center for Biodiversity Outcomes, Arizona State University, Tempe, Arizona 85287, USA.

e-mails: hugh.possingham@tnc.org; leah.gerber@asu.edu

1. Pimm, S. L. et al. Science 344, 1246752 (2014).

2. Balmford, A., Gaston, K. J., Blyth, S., James, A. \& Kapos, V. Proc. Natl Acad. Sci. USA 100, 1046-1050 (2003).

3. Waldron, A. et al. Nature 551, 364-367 (2017).

4. Cardillo, M. et al. Proc. R. Soc. B 275, 1441-1448 (2008).

5. Gregory, R. et al. Structured Decision Making: A Practical Guide to Environmental Management Choices (Wiley-Blackwell, 2012).

6. Hoffmann, M. et al. Science 330, 1503-1509 (2010)

7. Kerkvliet, J. \& Langpap, C. Ecol. Econ. 63, 499-510 (2007).

8. Butchart, S. H. et al. PLoS Biol. 2, e383 (2004).

9. Gerber, L. R. Proc. Natl Acad. Sci. USA 113, 3563-3566 (2016).

10.Joseph, L. N., Maloney, R. F. \& Possingham, H. P. Conserv. Biol. 23, 328-338 (2009).

11. McCarthy, D. P. et al. Science 338, 946-949 (2012).

12.McCarthy, M. A., Thompson, C. J. \& Garnett, S. T.

J. Appl. Ecol. 45, 1428-1435 (2008).

13.Wilson, H. B., Joseph, L. N., Moore, A. L. \&

Possingham, H. P. Ecol. Lett. 14, 886-890 (2011).

This article was published online on 25 October 2017.

\title{
A broadly protective antibody
}

\begin{abstract}
A single antibody uses multiple antiviral mechanisms to block the replication of influenza B viruses in mice and ferrets. The development could inform research into improved flu vaccines.
\end{abstract}

\section{PETER PALESE}

A $t$ the turn of the twentieth century, the immunologist Paul Ehrlich put forward the idea of 'magic bullets', which could be used in the fight against human diseases. Seventy years later, this concept received support, thanks to technology that enabled the production of antibodies designed to target almost any antigen - molecular structures produced by cells and viruses. In 2016, five of the eight top-grossing prescription drugs were monoclonal antibodies (those that bind to only one site on an antigen), which target viruses, bacteria, toxins and proteins (go.nature.com/2yidu1o). Writing in Science Translational Medicine, Shen et al. ${ }^{1}$ describe a monoclonal antibody that, in animals, potently cross-protects against all influenza B viruses tested. Such an antibody could protect humans against influenza B and could inform the design of broadly protective flu vaccines.

Seasonal outbreaks of influenza A and B strains are estimated to be responsible for the death of up to 50,000 people in the United States each year (see go.nature.com/2ao6jua). This disease burden is likely to remain steady in the near future, because current commercial flu vaccines are suboptimal. The occasional emergence of pandemic strains of the influenza A virus adds an extra element of unpredictability. Typically, seasonal and pandemic influenza A viruses pose the greatest threat to the population - but influenza B can also be associated with high mortality, especially in infants and children. 


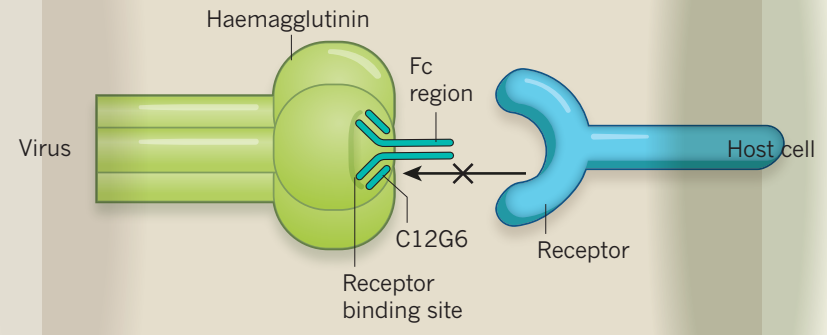

Figure 1 | Antibody protection against influenza B viruses. Shen $\mathrm{et} \mathrm{al.}{ }^{1}$ have created an antibody, dubbed C12G6, that can protect mice and ferrets against multiple strains of influenza B virus. a, C12G6 binds to an evolutionarily conserved region of the head of the viral glycoprotein haemagglutinin, thereby blocking haemagglutinin binding to receptors b Immune responses activated

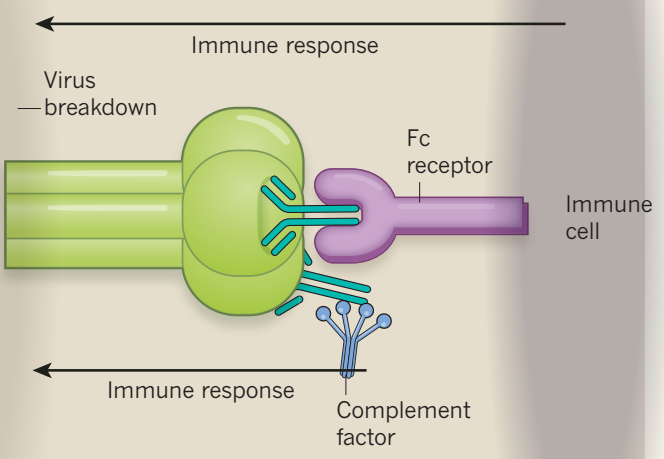

on host cells and so preventing virus replication. b, Unusually, C12G6 also seems to exert antiviral effects through its $\mathrm{Fc}$ region. This region binds to Fc receptors on immune cells, activating the cells to destroy the invading virus, and interacts with extracellular immune proteins called complement factors, again leading to virus breakdown.
Flu viruses dock to host cells through a glycoprotein called haemagglutinin, which binds to host-cell receptors. Antibodies that bind to haemagglutinin to prevent this docking can therefore protect animals and humans infected with flu viruses. Theoretically, treatment with such antibodies could both prevent and treat flu by providing people with immediate, short-term protection (passive immune protection).

Currently, circulating influenza B viruses belong to either the Victoria or the Yamagata lineage - the two split in the 1980s from an earlier precursor (see go.nature.com/2hivvpf). Antibodies that would protect against variants of both lineages are highly desirable. Three groups $^{2-4}$ have previously reported human monoclonal antibodies that bind to specific sites on $B$ virus haemagglutinins to provide protection against multiple strains of $B$ virus in mice. Shen et al. have now built on these findings.

The authors immunized mice with live influenza B viruses from both lineages to trigger antibody production by the animals' immune systems. They isolated hundreds of monoclonal antibodies produced from eight immunization regimens and analysed the ability of each antibody to block the attachment of influenza B viruses to cells (determined by measuring the level of haemagglutination - the clumping of red blood cells and viral particles bound together through haemagglutinin). The researchers selected the best antibody, dubbed 12G6, and combined the antigen-binding region of this antibody with the portion of a human IgG1 antibody, called the $\mathrm{Fc}$ region, that can bind to receptors on host immune cells to activate the human innate immune system. The group used the resulting chimaeric antibody, which they named C12G6, for all further studies.

Shen et al. demonstrated that C12G6 is a broadly protective antibody. C12G6 exhibited

haemagglutination-inhibition activity against all 18 influenza B viruses that the researchers tested and showed higher potency than the previous cross-protecting antibodies ${ }^{2-4}$. Importantly, C12G6 protected mice and ferrets against influenza $\mathrm{B}$ viruses when administered preventively, as well as providing excellent therapeutic effects when administered after viral infection.

Typically, an antibody that acts to inhibit the attachment of specific flu viruses to the host cell binds to a highly variable region on haemagglutinin's 'head' region. C12G6 is different, in that it binds to an evolutionarily conserved docking point on the head near the receptor-binding site. The group showed that

\section{“C12G6 protected mice and ferrets against influenza B viruses when administered preventively."} binding of C12G6 to this site interferes with the attachment of all $\mathrm{B}$ virus strains (not only a specific variant) to host cells, providing an explanation for the antibody's broad activity (Fig. 1a).

Next, Shen et al. showed that C12G6 can inhibit influenza B virus replication by not only neutralizing the virus, but also activating the antiviral activities of the host's immune system through Fcmediated mechanisms (Fig. 1b). In particular, C12G6 shows complement-dependent cytotoxicity, in which binding of the antibody's Fc region by immune-system proteins called complement factors triggers an extracellular signalling cascade that leads to the degradation of the virus. C12G6 also stimulates antibody-dependent cell-mediated cytotoxicity (ADCC), in which Fc receptors on immune cells are activated by antibody binding, triggering a direct immune response from the cell that again leads to virus destruction and elimination.
The ability of C12G6 to both block haemagglutination of host cells by a virus and elicit ADCC is highly unusual. It has previously been reported ${ }^{5,6}$ that haemagglutinationinhibiting antibodies block optimal activation of immune cells and so do not induce ADCC - more work is needed to explain this discrepancy.

What is next to be discovered? First, it has not yet been proved that $\mathrm{C} 12 \mathrm{G} 6$ is protective in humans. Second, even if it is effective, passive immunization against flu (especially influenza B) may be of limited value. Because of the short duration of protection and high cost of antibody therapeutics, this approach might not be as cost effective as seasonal vaccines. However, it could be useful for certain groups of people who are at risk of exposure, including health-care workers, emergency personnel and the military, because of the ability of broadly protective antibodies to be effective against multiple influenza variants.

Finally, it will be valuable to find out whether the head of a B virus haemagglutinin can be developed for use as a vaccine. To be of use, the haemagglutinin head region used would need to induce the human immune system to produce high titres of antibodies that have properties similar to those of C12G6, and provide long-lasting and broad immune protection. If successful, such a vaccine could be transformative.

Peter Palese is in the Department of Microbiology, Icahn School of Medicine at Mount Sinai, New York, New York 10029,

USA.

e-mail:peter.palese@mssm.edu

1. Shen, C. et al. Sci. Transl. Med. 9, eeam5752 (2017)

2. Yasugi, M. et al. PLOS Pathog. 9, e1003150 (2013).

3. Dreyfus, C. et al. Science 337, 1343-1348 (2012).

4. Chai, N. et al. Nature Commun. 8, 14234 (2017).

5. Leon, P. E. et al. Proc. Natl Acad. Sci. USA 113 E5944-E5951 (2016).

6. Cox, F. et al. Front. Immunol. 7, 399 (2016). 